\title{
Primary Total Knee Arthroplasty for Simple Distal Femoral Fractures in Elderly Patients with Knee Osteoarthritis
}

\author{
Nam-Yong Choi, $\mathrm{MD}^{1}$, Jong-Min Sohn, $\mathrm{MD}^{2}$, Sung-Gil Cho, $\mathrm{MD}^{1}$, Seung-Chan Kim, $\mathrm{MD}^{3}$, and Yong In, $\mathrm{MD}^{3}$ \\ ${ }^{1}$ Department of Orthopaedic Surgery, St. Paul's Hospital, Seoul; ${ }^{2}$ Department of Orthopaedic Surgery, Incheon St. Mary's Hospital, Incheon; ${ }^{3}$ Department of \\ Orthopaedic Surgery, Seoul St. Mary’s Hospital, The Catholic University of Korea College of Medicine, Seoul, Korea
}

\begin{abstract}
Purpose: Primary total knee arthroplasty (TKA) can be an alternative method for treating distal femoral fractures in elderly patients with knee osteoarthritis. The purpose of this study was to evaluate the clinical and radiographic results in patients with knee osteoarthritis who underwent TKA with the Medial Pivot prosthesis for distal femoral fractures.

Materials and Methods: Eight displaced distal femoral fractures in 8 patients were treated with TKA using the Medial Pivot prosthesis and internal fixation. The radiographic and clinical evaluations were performed using simple radiographs and Hospital for Special Surgery (HSS) knee scores during a mean follow-up period of 49 months.

Results: All fractures united and the mean time to radiographic union was 15 weeks. The mean range of motion of the knee joint was $114.3^{\circ}$ and the mean HSS knee score was 85.1 at the final follow-up.

Conclusions: Based on the radiographic and clinical results, TKA with internal fixation can be considered as an option for the treatment of simple distal femoral fractures in elderly patients who have advanced osteoarthritis of the knee with appropriate bone stock.
\end{abstract}

Keywords: Knee, Distal femoral fracture, Osteoarthritis, Arthroplasty, Medial Pivot

\section{Introduction}

Distal femoral fractures in the elderly usually occur as a result of low energy trauma, and are difficult to treat because of osteoporosis and pre-existing osteoarthritis ${ }^{1,2)}$. Traditional methods for internal fixation of distal femoral fractures have included the 95-degree angled blade plate fixation, dynamic condylar screw fixation and retrograde nailing ${ }^{3-7)}$. More recently, anatomic

Received March 20, 2013; Revised (1st) May 19, 2013; (2nd) July 8, 2013; Accepted July 15, 2013

Correspondence to: Yong In, MD

Department of Orthopaedic Surgery, Seoul St. Mary's Hospital, The Catholic University of Korea College of Medicine, 222 Banpo-daero, Seocho-gu, Seoul 137-701, Korea

Tel: +82-2-2258-2838, Fax: +82-2-535-9834

E-mail: iy1000@catholic.ac.kr

This is an Open Access article distributed under the terms of the Creative Commons Attribution Non-Commercial License (http://creativecommons.org/licenses/by-nc/3.0/) which permits unrestricted non-commercial use, distribution, and reproduction in any medium, provided the original work is properly cited. locking condylar plates have been developed to treat these fractures ${ }^{8,9)}$. However, poor knee function, prolonged confinement to bed and high mortality rate have been noted even after operative treatment in elderly patients ${ }^{1,2,5,10)}$.

Primary total knee arthroplasty (TKA) has been advocated as a treatment modality in patients with distal femoral fractures who already have a painful arthritic knee ${ }^{1,10-15)}$. Most of them have been treated using a hinged prosthesis ${ }^{1,10-12,14)}$. However, there are concerns about the high rate of loosening and mechanical failure of this type of prosthesis ${ }^{10,13)}$. Yoshino et al. ${ }^{15)}$ reported on 3 cases of posterior-stabilized (PS) TKAs. But, PS femoral component also requires an intercondylar box cut for cam and post mechanism and this procedure may affect the fracture stability in case of juxta articular fractures ${ }^{13)}$. In et al. ${ }^{13)}$ treated 3 cases using cruciate-retaining (CR) augmentable total knee implants with stem extension.

The Medial Pivot Knee (Wright Medical Technology Inc., Arlington, TN, USA) was developed specifically to replicate normal knee kinematics and reduce polyethylene wear by providing more conforming surface shapes ${ }^{16,17)}$. Posterior cruciate ligament www.jksrr.org 
(PCL) can either be retained or sacrificed during the soft tissue balancing procedure without femoral box cutting ${ }^{18)}$. Femoral stem can be easily extended with minimal constraint as per the requirement. We are of the opinion that the Medial Pivot Knee offers advantages of preservation of femoral bone stock and flexibility in the decision of PCL treatment with the use of the same component in TKA for distal femoral fractures.

The purpose of this study was to evaluate the clinical and radiographic results in patients with knee osteoarthritis who underwent TKA with the Medial Pivot prosthesis for distal femoral fractures.

\section{Materials and Methods}

Between January 2006 and December 2009, 8 displaced distal femoral fractures in 8 patients were treated with TKA using the Medial Pivot prosthesis. All patients were females and the average age at the time of surgery was 76.8 years (range, 65 to 89 years) (Table 1). All fractures had occurred after fall to the ground during walking. All patients had been treated conservatively for advanced osteoarthritis in the affected knee and considering TKA. Among them, 2 patients had been scheduled for TKA. No patient had a history of previous knee surgery. We explained the treatment modalities and possible complications. All patients were scheduled for TKA for the treatment of distal femoral fractures and knee osteoarthritis at the same time and gave their written informed consent. The Arbeitsgemeinschaft für Osteosynthesefragen/Orthopaedic Trauma Association (AO/OTA) classification ${ }^{19)}$ was used to classify the fractures. All fractures were caused by low-energy trauma. Three patients had type A or extra articular fractures (two cases of A1 fractures and one case of A2 fracture). Two patients had type B or condylar fractures (two cases of B2 fractures). Three patients had type $\mathrm{C}$ or supracondylar fractures with an intercondylar extension (three cases of $\mathrm{C} 1$ fractures).

All operations were performed under general endotracheal anesthesia. Patients were positioned supine on a radiolucent operation table. A standard midline skin incision in conjunction with medial parapatellar approach was used. According to the fracture type, the incision was extended proximally. Preliminary deep medial collateral ligament (MCL) release was performed at the tibial attachment site using a periosteal elevator in all patients. Displaced fractures were addressed first with reduction and application of bone clamps to the femoral shaft and a pelvic bone clamp to the condyles. Temporary Kirschner wire fixation was performed to maintain the fracture reduction during TKA procedure. Distal femoral cut was performed using the intramedullary femoral cutting guide. Anterior and posterior condylar cuts and chamfer cuts were made while holding the clamps. A proximal tibial cut was made using the extramedullary cutting guide. Femoral stems were used in 6 patients depending on the fracture stability. PCL was resected to achieve soft tissue balance in 5 patients who had a tight flexion gap after bone cutting. Flexion-extension gap balance was achieved in all patients. Femoral and tibial components were implanted with cement. To promote fracture union, bone cement was not filled into the femoral medullary canal. Additional fixation was performed using Kirschner wires, Dall-Miles cables, and screws depending on the fracture stability after prosthesis implantation. The mean tourniquet time was 76 minutes (range, 55 to 95 minutes) and the mean blood loss during operation was $365 \mathrm{~mL}$ (range, 220 to $500 \mathrm{~mL}$ ).

After surgery, a long leg splint was applied and it was worn for

Table 1. Study Population Data $(n=8)$

\begin{tabular}{|c|c|c|c|c|c|c|c|c|}
\hline Case & Age (yr) & $\begin{array}{c}\text { Fracture } \\
\text { type }\end{array}$ & $\begin{array}{l}\text { Use of } \\
\text { stem }\end{array}$ & $\begin{array}{c}\text { PCL } \\
\text { sacrifice }\end{array}$ & Additional fixation used & $\begin{array}{c}\text { Time to } \\
\text { union (wk) }\end{array}$ & $\begin{array}{c}\text { Range of } \\
\text { motion }\left({ }^{\circ}\right) \text { at } 2 \mathrm{yr}\end{array}$ & $\begin{array}{c}\text { HSS score } \\
\text { at } 2 \mathrm{yr}\end{array}$ \\
\hline 1 & 72 & A1 & Yes & No & Screws $(\times 2)$ & 12 & $0-125$ & 90 \\
\hline 2 & 86 & $\mathrm{C} 1$ & Yes & Yes & Screw, wires $(\times 2)$, cables $(\times 2)$ & 20 & $5-120$ & 88 \\
\hline 3 & 72 & $\mathrm{C} 1$ & Yes & Yes & Screws $(\times 2)$, cables $(\times 3)$, K-wire & 16 & $0-120$ & 84 \\
\hline 4 & 89 & $\mathrm{~B} 2$ & No & Yes & Screws $(\times 2)$, cable & 12 & $0-110$ & 82 \\
\hline 5 & 87 & $\mathrm{~B} 2$ & No & No & Screws $(\times 2), \mathrm{K}$-wires $(\times 2)$ & 16 & $0-110$ & 80 \\
\hline 6 & 71 & A1 & Yes & Yes & Screw & 16 & $0-120$ & 88 \\
\hline 7 & 73 & $\mathrm{C} 1$ & Yes & No & Screw & 16 & $10-120$ & 90 \\
\hline 8 & 65 & $\mathrm{~A} 2$ & Yes & Yes & Screws $(\times 3)$, wire & 12 & $5-110$ & 79 \\
\hline
\end{tabular}

All patients were female and they had Kellgren-Lawrence grade IV osteoarthritis on injured knee.

The mechanism of injury was fall down in all cases.

PCL: posterior cruciate ligament, HSS: Hospital for Special Surgery, K-wire: Kirschner wire. 
1 to 6 weeks depending on the fracture stability. On the second postoperative day, the drain was removed and passive range of motion exercise was performed twice daily for 30 minutes each time using the continuous passive motion (CPM) machine to $90^{\circ}$ of knee flexion. Partial weight bearing using crutches was allowed from 1 to 6 weeks after surgery.

Clinical and radiographic evaluations were made at 6 weeks and 3 months after surgery. If there was no evidence of bony union at the postoperative 3-month visit, the patient was followed up monthly till the fracture united. The patients were followed up for 1 year after surgery and then yearly thereafter. Clinical results were evaluated using Hospital for Special Surgery (HSS) knee scores $^{20)}$ and range of motion. Radiographic evaluation was performed using anteroposterior (AP), lateral, and merchant radiographic views of the knee. Evidence of fracture union, positioning and maintenance of prosthesis, and the lower extremity alignment were carefully examined. Fracture union was assessed using the findings of cortical or trabecular bridging and disappearance of fracture lines.

\section{Results}

The mean follow-up period was 49 months (range, 17 to 62 months). There were no cases of infection or perioperative death. All fractures united and the mean time to radiographic union was 15 weeks (range, 12 to 20 weeks). The mean femorotibial angle at the final follow-up was valgus $5.7^{\circ}$ (range, valgus $4^{\circ}$ to $8^{\circ}$ ). The Medial Pivot prosthesis was found to be easily adaptable for stem extension without additional bone cutting. Three patients with supracondylar fractures (AO/OTA classification A) (Fig. 1) and 3 patients with supra-intercondylar fractures (AO/OTA classification C) (Fig. 2) were treated using a stem extension. Two
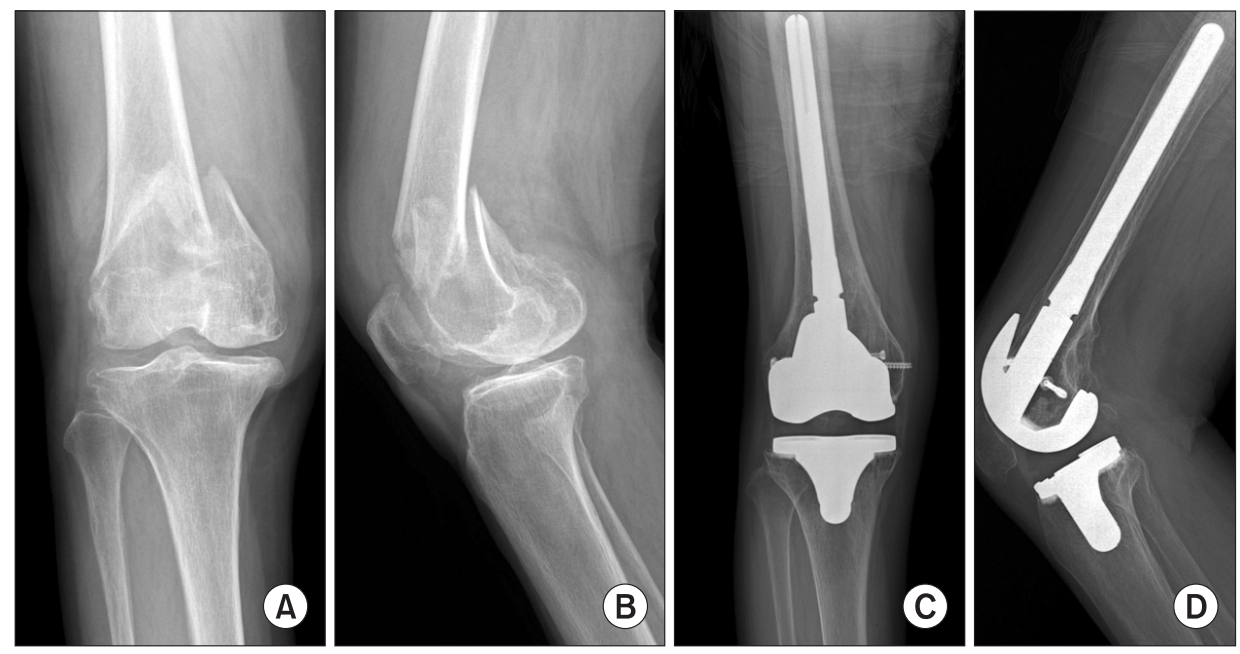

Fig. 1. Preoperative radiographs (A, B) of a 72-year-old female patient show an Arbeitsgemeinschaft für Osteosynthesefragen/ Orthopaedic Trauma Association type A1 distal femoral fracture. Medial Pivot total knee arthroplasty was performed with the use of a stem. Postoperative radiographs (C, D) show bony union with a well-maintained prosthesis (case 1).
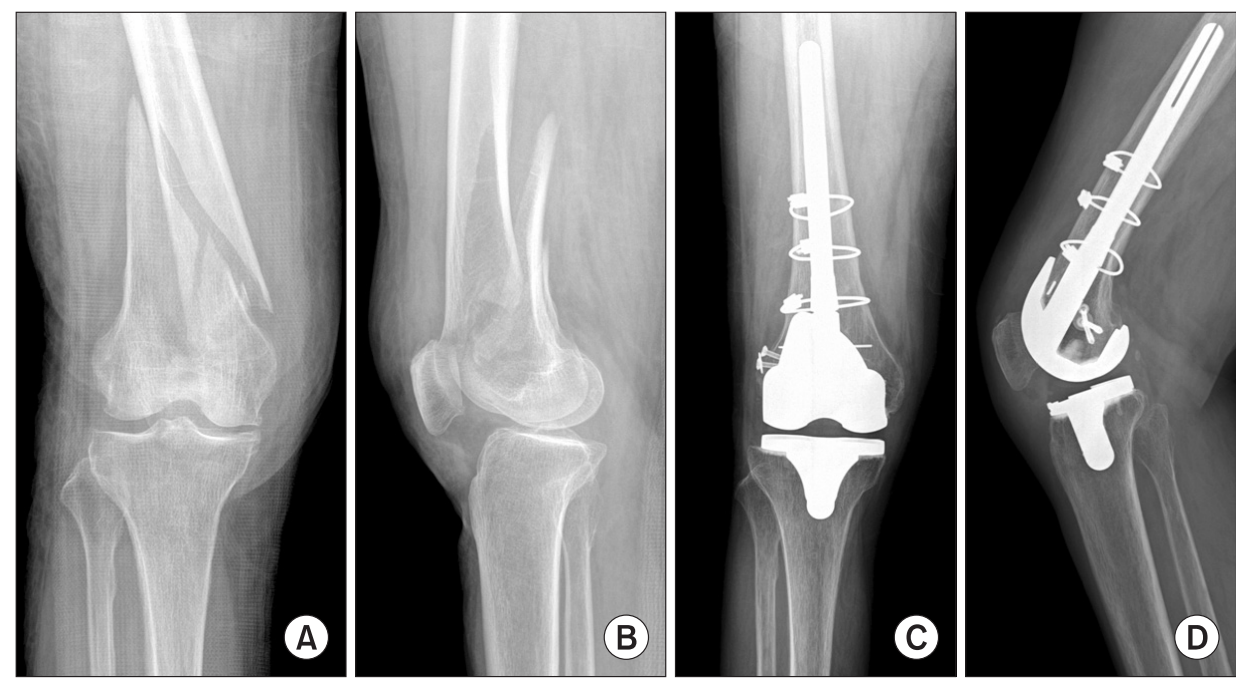

Fig. 2. Preoperative radiographs (A, B) of a 72-year-old female patient show an Arbeitsgemeinschaft für Osteosynthesefragen/ Orthopaedic Trauma Association type C1 distal femoral fracture. Medial Pivot total knee arthroplasty was performed with the use of a stem. Additional fixations were performed using Dall-Miles cables and screws. Postoperative radiographs (C, D) show bony union and the prosthesis is in good alignment with the stem (case 3). 

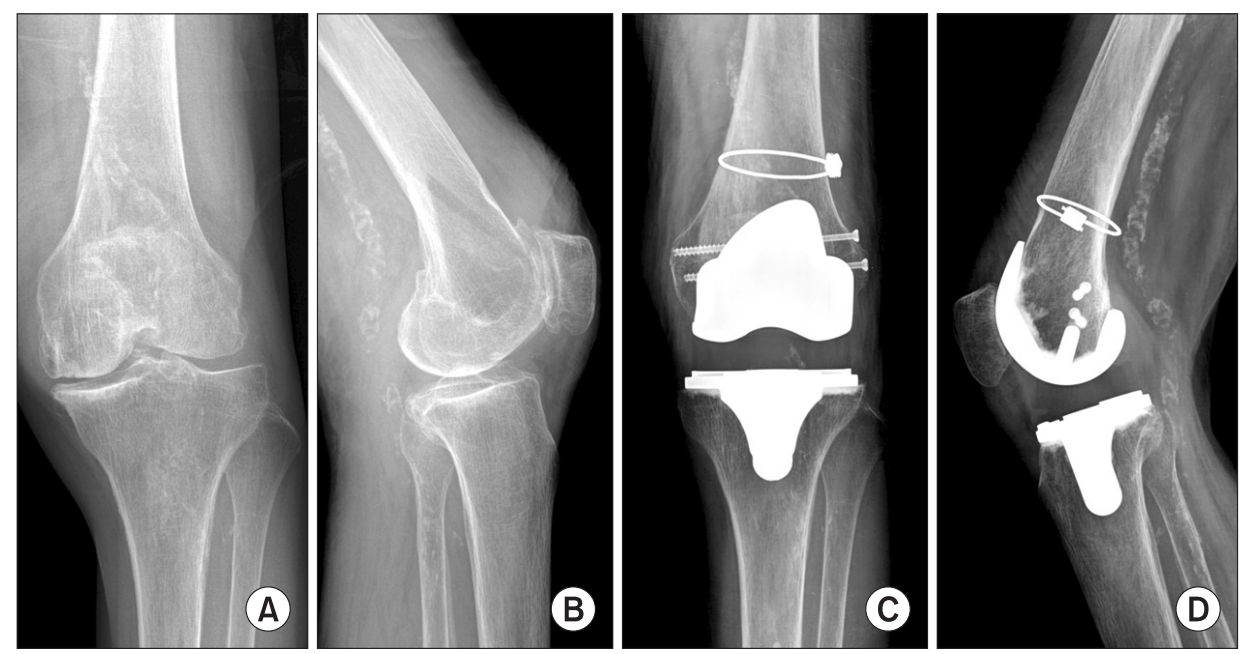

Fig. 3. Preoperative anteroposterior and lateral radiographs (A, B) of an 89-year-old female patient show an Arbeitsgemeinschaft für Osteosynthesefragen/Orthopaedic Trauma Association classification type B2 distal femoral fracture. Medial Pivot total knee arthroplasty was performed without the use of a stem. Postoperative radiographs (C, D) show bony union with a well-maintained prosthesis (case 4).
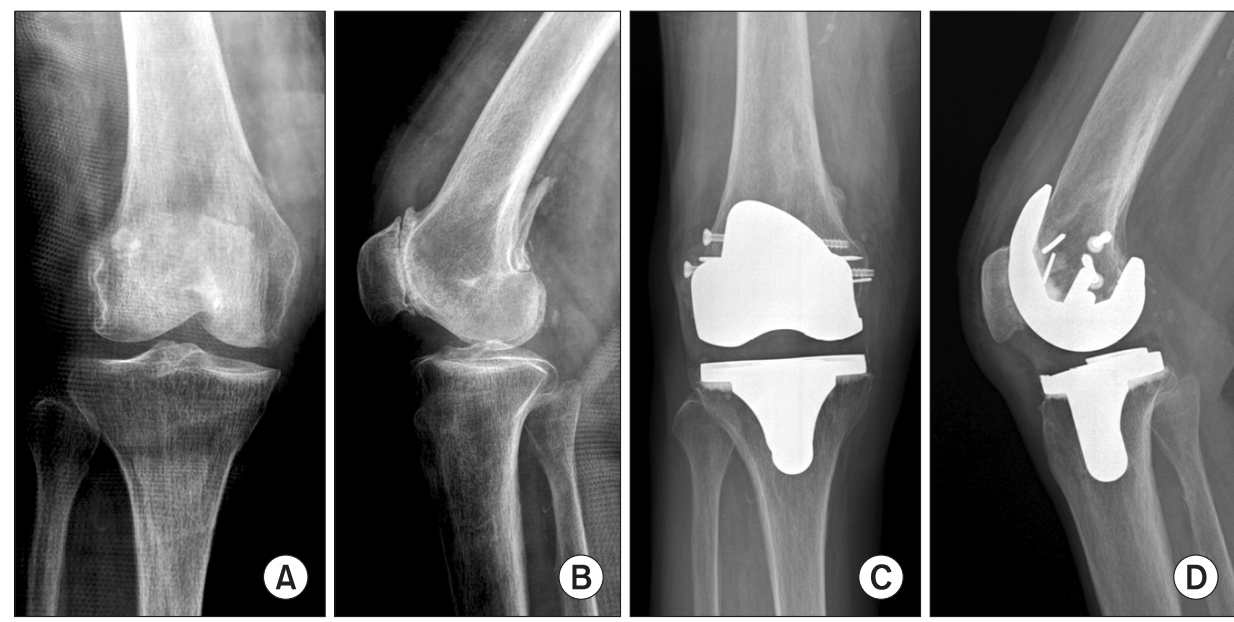

Fig. 4. Preoperative anteroposterior and lateral radiographs of an 87-year-old female patient (A, B) show an Arbeitsgemeinschaft für Osteosynthesefragen/Orthopaedic Trauma Association classification type B2 distal femoral fracture. Medial Pivot total knee arthroplasty was performed without the use of a stem. Postoperative radiographs (C, D) show bony union with a well-maintained prosthesis (case 5).

patients who had condylar fractures (AO/OTA classification B) were treated without using a stem extension (Fig. 3, 4). There was no angular deformity, malunion, or shortening on the AP and lateral knee radiographs of all cases. The components were well maintained without evidence of loosening. All knees were stable anteroposteriorly and mediolaterally regardless of PCL retention or sacrifice. The mean postoperative flexion contracture of the knee joint was $2.5^{\circ}$ (range, $0^{\circ}$ to $10^{\circ}$ ). At the final follow-up, the mean range of motion of the knee joint was $114.3^{\circ}$ (range, $105^{\circ}$ to $125^{\circ}$ ) and the mean HSS knee score was 85.1 (range, 79 to 90).

\section{Discussion}

In the present study, TKA was a viable option for the treatment of distal femoral fractures in the elderly who have knee osteoarthritis. Getting relief of the arthritic pain and improvement of the knee function at the time of fracture treatment was considered as the advantages of TKA over osteosynthesis in our patients. TKA can be performed after bony union through the osteosynthesis procedure in these patients. However, the elderly patients should be under anesthesia for additional procedures. It also costs money and time. In our patients, all fractures united with good range of motion of the knee and HSS scores. The advantages of the Medial Pivot prosthesis that we discovered were ease of stem extension without additional bone resection and flexibility in flexion gap balancing with the use of the same components.

Distal femoral fractures usually have a bimodal age distribution $^{1)}$. In the younger age group, comminuted fractures are usually caused by traffic accidents and are best treated with anatomical reduction and internal fixation ${ }^{2,4)}$. In contrast, distal femoral fractures in elderly patients are often simple fractures and are caused by minor trauma ${ }^{1,2,4)}$. Thin cortices, osteoporosis, a wide intramedullary canal, and osteoarthritis of the knee make stable fixation difficult to achieve particularly in infirm women who 
have medical problems ${ }^{1,9)}$. The patients included in this study were the typical cases of distal femoral fractures. All patients were diagnosed with osteoporosis. Seven of 8 patients were more than 70 years of age. Only 1 patient had a mechanically unstable fracture of the distal femur (AO/OTA classification A2, A3, C2, and C3 ${ }^{9,19)}$. However, surgical decision for TKA cannot be made with only radiographic evidence of knee osteoarthritis in these elderly patients. All patients in our study had been suffering from the arthritic pain of the knee and wanted to address the knee arthritis at the same time if possible. If the elderly people are in a highvelocity accident, mechanically unstable distal femoral fractures with severe comminution would develop certainly. In such a case, a standard TKA with stem extension might be impractical and other treatment options using a hinged knee prosthesis or constrained prosthesis would be a better alternative ${ }^{13)}$. However, most of the distal femoral fractures in the elderly patients are stable fractures and can be treated with a standard TKA prosthesis.

The Medial Pivot prosthesis is a newer concept design ${ }^{16,17)}$. AP stability is achieved by the ball and socket configuration of the medial compartment. Translation in the lateral compartment is unrestricted. It does not roll back as in the post and cam mechanism of the PS prosthesis. In vivo fluoroscopic analysis of the Medial Pivot prosthesis has shown that the medial femoral condyle remains fully constrained and posterior translation occurs in the lateral compartment, as called for by the design ${ }^{16)}$. The femoral preparation procedure only requires condylar and chamfer cuts regardless of whether or not the stem is extended. We thought that the Medial Pivot prosthesis has considerable merits comparable to those of the $\mathrm{CR}$ augmentable femoral component for its use in TKA for the treatment of distal femoral fractures in the elderly who have knee osteoarthritis ${ }^{13)}$.

Technical considerations include the decision regarding the use of a femoral stem according to the fracture stability. In our study population, two patients had AO/OTA type B2 fractures. After bone cuts and trial component insertion, we felt that the fracture was mechanically stable. Hence, we decided not to use the femoral stem. As a result of implanting the femoral component with cement and performing additional fixation with screws and cables, good fracture stability was achieved. Both patients were treated uneventfully. However, we believe that the femoral stem should be used for mechanically unstable fractures. Other considerations include balancing the PCL depending on the flexion gap. The Medial Pivot prosthesis is different from the CR augmentable implant since it allows for flexibility in the decision of PCL treatment. With the use of the Medial Pivot prosthesis, PCL can be excised depending on the flexion gap tightness without violating the joint stability. In our study, PCL was sacrificed in 5 patients who had flexion gap tightness. Good fracture stability was achieved in all patients at the final follow-up regardless of PCL resection.

This study has several limitations. First, there was a small number of patients. Second, there was no control group for the comparison. If a retrospective cohort study could have been performed with a large number of patients, it would have shown more decisive conclusions than those of this study. However, distal femoral fractures are relatively uncommon in the elderly and this study included a 4-year consecutive case series at a University Medical Center. Long-term durability of the implant is another concern. The longevity of the insert and component loosening are major possible causes of failure of the implant. Longer followup is required to determine the true efficacy of this type of treatment. Nevertheless, we think that the longevity of the prosthesis in the patients included in this study would be the same as that in the patients who underwent primary Medial Pivot TKA for osteoarthritis of the knee.

\section{Conclusions}

Based on the clinical and radiographic results, TKA with the Medial Pivot prosthesis can be considered as an option for the treatment of minimally comminuted distal femoral fractures in elderly patients who have advanced osteoarthritis of the knee with appropriate bone stock.

\section{Conflict of Interest}

No potential conflict of interest relevant to this article was reported.

\section{References}

1. Bell KM, Johnstone AJ, Court-Brown CM, Hughes SP. Primary knee arthroplasty for distal femoral fractures in elderly patients. J Bone Joint Surg Br. 1992;74:400-2.

2. Marks DS, Isbister ES, Porter KM. Zickel supracondylar nailing for supracondylar femoral fractures in elderly or infirm patients: a review of 33 cases. J Bone Joint Surg Br. 1994;76:596-601.

3. Higgins TF, Pittman G, Hines J, Bachus KN. Biomechanical analysis of distal femur fracture fixation: fixed-angle screw-plate construct versus condylar blade plate. J Orthop Trauma. 2007;21:43-6. 
4. Mize RD, Bucholz RW, Grogan DP. Surgical treatment of displaced, comminuted fractures of the distal end of the femur. J Bone Joint Surg Am. 1982;64:871-9.

5. Muckley T, Wahnert D, Hoffmeier KL, von Oldenburg G, Frober R, Hofmann GO. Internal fixation of type-C distal femoral fractures in osteoporotic bone: surgical technique. J Bone Joint Surg Am. 2011;93 Suppl 1:40-53.

6. Petsatodis G, Chatzisymeon A, Antonarakos P, Givissis P, Papadopoulos P, Christodoulou A. Condylar buttress plate versus fixed angle condylar blade plate versus dynamic condylar screw for supracondylar intra-articular distal femoral fractures. J Orthop Surg (Hong Kong). 2010;18:35-8.

7. Schatzker J, Mahomed N, Schiffman K, Kellam J. Dynamic condylar screw: a new device. A preliminary report. J Orthop Trauma. 1989;3:124-32.

8. Kregor PJ, Stannard JA, Zlowodzki M, Cole PA. Treatment of distal femur fractures using the less invasive stabilization system: surgical experience and early clinical results in 103 fractures. J Orthop Trauma. 2004;18:509-20.

9. Weight M, Collinge C. Early results of the less invasive stabilization system for mechanically unstable fractures of the distal femur (AO/OTA types A2, A3, C2, and C3). J Orthop Trauma. 2004;18:503-8.

10. Appleton P, Moran M, Houshian S, Robinson CM. Distal femoral fractures treated by hinged total knee replacement in elderly patients. J Bone Joint Surg Br. 2006;88:1065-70.

11. Anderson SP, Matthews LS, Kaufer H. Treatment of juxtaarticular nonunion fractures at the knee with long-stem total knee arthroplasty. Clin Orthop Relat Res. 1990;(260):104-9.

12. Freedman EL, Hak DJ, Johnson EE, Eckardt JJ. Total knee replacement including a modular distal femoral component in elderly patients with acute fracture or nonunion. J Orthop Trauma. 1995;9:231-7.

13. In Y, Koh HS, Kim SJ. Cruciate-retaining stemmed total knee arthroplasty for supracondylar-intercondylar femoral fractures in elderly patients: a report of three cases. J Arthroplasty. 2006;21:1074-9.

14. Wolfgang GL. Primary total knee arthroplasty for intercondylar fracture of the femur in a rheumatoid arthritic patient: a case report. Clin Orthop Relat Res. 1982;(171):80-2.

15. Yoshino N, Takai S, Watanabe Y, Fujiwara H, Ohshima Y, Hirasawa Y. Primary total knee arthroplasty for supracondylar/condylar femoral fracture in osteoarthritic knees. J Arthroplasty. 2001;16:471-5.

16. Schmidt R, Komistek RD, Blaha JD, Penenberg BL, Maloney WJ. Fluoroscopic analyses of cruciate-retaining and medial pivot knee implants. Clin Orthop Relat Res. 2003;(410):13947.

17. Shakespeare D, Kinzel V, Ledger M. Achieving ligament stability and correct rotational alignment of the femur in knee arthroplasty: a study using the Medial Pivot knee. Knee. 2005;12:419-23.

18. Bae DK, Song SJ, Cho SD. Clinical outcome of total knee arthroplasty with medial pivot prosthesis a comparative study between the cruciate retaining and sacrificing. J Arthroplasty. 2011;26:693-8.

19. Fracture and dislocation compendium. Orthopaedic Trauma Association Committee for coding and classification. J Orthop Trauma. 1996;10 Suppl 1:1-154.

20. Insall JN, Ranawat CS, Aglietti P, Shine J. A comparison of four models of total knee-replacement prostheses. J Bone Joint Surg Am. 1976;58:754-65. 